\title{
Beca docente en el Perú: una experiencia de colaboración público privada, más allá de la responsabilidad social universitaria
}

\author{
INÉS RUIZ DE CASTILLA* \\ Pontificia Universidad Católica del Perú \\ Recibido el 24-02-2017; primera evaluación el 18-12-2017; \\ segunda evaluación el 20-02-2018, aceptado el 22-02-2018
}

\section{RESUMEN}

Las organizaciones universitarias, como la Pontificia Universidad Católica del Perú (PUCP), han asumido con responsabilidad la labor de conexión entre la universidad y la sociedad y promueven el proceso de convenios público privados, como el desarrollado con el Programa Nacional de Becas y Crédito Educativo del Perú (Pronabec), para favorecer y desarrollar su experiencia en capacitación de docentes de la Carrera Pública Magisterial con el fin de mejorar los rendimientos docentes y así contribuir a la calidad educativa de los aprendizajes.

La Beca Docente Presidente de la República contribuyó en la formación personal y académica de docentes de la carrera pública magisterial peruana, quienes, a través de un concurso de mérito, accedieron a conformar una elite de profesionales que se benefició de la valiosa contribución que aportaron las universidades participantes del proyecto, en especial, la Pontificia Universidad Católica del Perú, que puso a disposición de los alumnos becarios de Maestría, las competencias y capacidades que exige la educación y sociedad actuales. En ese propósito, se destinaron esfuerzos y recursos más allá de lo estipulado en los Convenios marco firmados, asumiendo la misión encomendada como un proceso propio de responsabilidad social universitaria (RSU) en una positiva experiencia que superó, largamente, las expectativas proyectadas, en beneficio de la calidad educativa de amplios sectores del país.

Palabras clave: Universidad, carrera pública magisterial, responsabilidad social universitaria, compromiso social, sociedad, Beca Presidente de la República, necesidades y problemas sociales, estudios de maestría, posgrado, docentes, calidad educativa, colaboración público-privada.

\footnotetext{
* Licenciada en Educación por la Facultad de Educación de la PUCP, Lima. Máster en TIC por las Universidades Carlos III de Madrid, Autónoma de Barcelona y Alicante. Gestora de la Maestría en Integración e Innovación Educativa de las TIC, convenio PUCP - Pronabec (20142015). Contacto: nes.ruizdecastilla@pucp.edu.pe
} 
Scholarship of schoolteachers in Peru: an experience of public-private collaboration, beyond the University Social Responsibility

\section{Abstract}

The current university organizations have assumed responsibility for the connection between the university and society and promote the process of public-private agreements, as developed between the Pontifical Catholic University of Peru (PUCP) and the National Scholarship and Educational Credit Program (PRONABEC) of the Government of Peru, in order to promote, and develop, their experience in the training of teachers of the Public School Magisterial in order to improve the teaching performance and the quality of learning in students. This article presents some guidelines developed during the execution of the President of the Republic Scholarship (2014-2015) and which in various ways explain the vocation of service and commitment to educational development, which PUCP has been carrying out since its founding as a university institution. In this regard, the University devoted resources and resources beyond what was agreed in the signed agreements, assuming the mission as a proper process of University Social Responsibility (USR) in a positive experience that far exceeded the expectations projected for the benefit of educational quality of teachers and students of the country.

Keywords: University, Public Teaching Career, University Social Responsibility, Social Commitment, Society, social needs and problems, Master's studies, postgraduate, teachers, educational quality, private-public collaboration.

Bolsa de estudos universitários para professores de escola no Perú: uma experiência de colaboraçáo público-privada, além da responsabilidade social universitária

\section{Resumo}

Organizaçóes universitários atuais, assumiram a responsabilidade pelo trabalho de ligação entre a universidade ea sociedade e promover o processo de parcerias público-privadas como desenvolvido entre a Pontifícia Universidade Católica do Peru (PUCP) com o Programa Nacional de Bolsas de Estudo e Crédito Educativo do Peru (PRONABEC) do Governo do Peru, para promover e desenvolver a sua experiência na formação de professores de Educador Pública, a fim de melhorar o desempenho educacional e qualidade do ensino da aprendizagem dos alunos. Este artigo apresenta algumas orientaçóes desenvolvidas durante a execução do Bolsa de estudos Presidente da República (2014-2015) e de várias maneiras explicar a vocação de serviço e compromisso com o desenvolvimento educacional, a PUCP tem vindo a fazer desde a sua fundaçáo como universidade. Nesse propósito da universidade, esforços e recursos para além do que foi acordado nos acordos 
assinados dedicado, assumindo a missão de si um processo de Responsabilidade Social Universitária (RSU) em uma experiência positiva que excedeu longa expectativas projetadas para o benefício da qualidade da educação de professores e alunos em todo o país.

Palavras-chave: universidade, carreira de ensino público, responsabilidade social universitária, compromisso social, sociedade, Bolsa de estudos do Presidente da República, necessidades e problemas sociais, mestrados, pós-graduação, professores, qualidade educacional, colaboração público-privada.

\section{INTRODUCCIÓN}

El concepto de universidad, se contrapone a lo exclusivo y elitista, más bien, se proyecta a ser general, universal y universalizante, de allí el término Universidad. Es, entonces discorde a circunscribir el conocimiento, se opone a la formación de grupos académicos reducidos o cerrados, en contraste, establece asociaciones o comunidades de docentes, investigadores y alumnos que comparten como motivación común, el estudio, la divulgación y la transmisión del conocimiento científico (Claramunt, 2002).

\section{Del SENTIDo social UNIVERSITARio a La RESPONSABILIDAD SOCIAL UNIVERSITARIA}

La universidad encuentra su sentido en la medida que se proyecta hacia la sociedad y hacia los ciudadanos. Acorde con la naturaleza social e histórica y con el compromiso que asume la institución universitaria con la sociedad en su conjunto, es que esta se proyecta en función de ella y de sus requerimientos más urgentes (Martinez, 2010).

La responsabilidad social, en su concepto más amplio, constituye una visión de serio compromiso con el entorno en el cual se desarrolla. Por lo tanto, presenta un conjunto de acciones que buscan el bien común, contiene elementos éticos muy importantes como la solidaridad, la colaboración, el genuino interés por el desarrollo comunitario, el progreso generalizado, entre otros aspectos (De la Calle, 2017).

De la responsabilidad social como concepto genérico se desprende la llamada responsabilidad social universitaria (RSU) que representa una respuesta concreta y directa a las necesidades y problemáticas que presenta el entorno en el cual ejerce su labor y función; de ninguna manera significa un pago, retribución o algo parecido (Vallaeys, 2017 y De la Red, 2009). 
Es, simplemente, el llegar al convencimiento de la importancia que tiene el compartir el conocimiento y hacer partícipe a la sociedad, en lo que puede aportar, el poder de transformación y progreso que puede ejercer en el desarrollo educativo, cultural, laboral y económico (De la Cuesta, 2010).

La Universidad, como institución social, toma este compromiso inherente a ella y establece lo mejor que tiene: los recursos humanos, la logística, los conocimientos y toda la experiencia para entender mejor a la sociedad y satisfacer sus necesidades (Martí Noguera y Martí Vilar, 2013). De esta manera, las aulas universitarias se convierten en foros de análisis de la realidad social y de las demandas de desarrollo humano y material que se necesita atender (Martínez de Carrasquero, 2008). La Universidad, por tanto, no está ajena a las necesidades de la población; las facultades universitarias tienen el deber de orientarse e implicarse más en el desarrollo de proyectos de desarrollo que beneficien a los sectores poblacionales relacionados con su ámbito académico (Gasca-Pliego y Olvera, 2011).

En dicho contexto, la Pontificia Universidad Católica del Perú (PUCP), desde un enfoque humanístico, cristiano y proyectado a la sociedad, desarrolla, en gran medida, una importante labor por la cual se conecta con la sociedad, se manifiesta y trabaja de la mano de ella para contribuir, desde su particular ámbito de acción, con la mejora de las condiciones de vida y el desarrollo social de las diversas comunidades del país (PUCP, 2017). La contribución se evidencia a través de la planificación de actividades académicas que se vinculan y enlazan con puntuales aspectos y ámbitos de atención social. Los alumnos de pregrado, por ejemplo, desde los primeros ciclos, tienen la posibilidad de ser activos partícipes de propuestas académicas proyectadas al desarrollo y bienestar social. Esto se ve reforzado gracias a que docentes y autoridades de la Universidad favorecen oportunidades cada vez más frecuentes a los estudiantes en formación para que se acerquen progresivamente al conocimiento de la realidad social y sus necesidades, sensibilizándose y comprometiéndose más con ella. La diversidad que descubre el Perú, tanto en geografía, lenguas y culturas, exige iniciativas de desarrollo comunitario que propongan no solo la ayuda directa sino también el asesoramiento y capacitación de las poblaciones, otorgándoles, así, las herramientas de conocimiento que posibiliten su mejor desarrollo personal y colectivo.

\section{La PUCP y SU PARTICIPACIÓN EN La BECa DOCENTE}

El compromiso que, desde su fundación, ha ejercido la PUCP en la sociedad peruana se ha plasmado en un programa sistemático que se viene desarrollando desde la DARS (Dirección Académica de Responsabilidad Social) 
(PUCP, 2017) a través de la cual viene desarrollando, de manera permanente, acciones concretas que conducen al establecimiento de un plan de acción para atender a diversos sectores de la población, consciente de que, como institución universitaria, debe implicarse, cada vez más, con la sociedad. En ese sentido, es que se constituyó como una de las instituciones educativas superiores participantes de la Propuesta de la Beca Presidente de la República (Pronabec, 2014), por medio de un Convenio Marco entre el Ministerio de Educación del Perú, a través del Programa Nacional de Becas (Pronabec), y cuatro prestigiosas universidades del país (Universidad de Piura, Universidad San Ignacio de Loyola, Universidad Peruana Cayetano Heredia y la Pontificia Universidad Católica del Perú) con el fin de desarrollar un programa de becas de estudios de posgrado destinado a docentes de la Carrera Pública Magisterial para la obtención del grado de magíster. La concepción de la propuesta, de por sí bastante ambiciosa, contemplaba el desplazamiento físico de los becarios docentes desde sus ciudades de origen a las sedes de las universidades para seguir sus estudios de posgrado de manera presencial y a dedicación exclusiva, con goce de haber y financiamiento del $100 \%$ de los costos académicos que exigía cada universidad, así como asignaciones mensuales para alimentación, transporte y alojamiento, con todo lo que ello representa, no solamente en términos de inversión financiera, sino, también, en aspectos logísticos y administrativos para asegurar, mediante coordinaciones directas con las Unidades de Gestión Educativa Local (UGEL) de cada región, puesto que las plazas que dejaban vacantes por el período de estudio de Maestría debían cubrirse con otros docentes.

La PUCP asumió la responsabilidad y, de manera decidida, se comprometió a disponer el recurso material y el elemento humano y académico para contribuir en esta propuesta bastante compleja por sus alcances, puesto que nunca antes había participado en algo semejante. Representó un reto importante en la medida que los alumnos becarios provenían de las tres regiones del país y, en la mayoría de casos, se iniciaban con una escasa, y hasta nula formación previa en procesos de investigación, lo cual significaba, en un primer momento, un gran escollo, pero que, luego, representó también una oportunidad para diseñar estrategias cada vez más acordes con las necesidades de los estudiantes. La clara visión social de la PUCP ubicó, a disposición de los estudiantes, todos los recursos y servicios necesarios para desarrollar con éxito su formación académica y personal. Desde esa perspectiva, se desarrollaron acciones que, desde el inicio de las actividades académicas, siempre convinieron en la misma dirección, que es transmitir a los alumnos becarios todo aquello que la Universidad posee: su infraestructura, la calidad formativa a través de sus docentes, asesores y tutores y, de esta forma, acompañar a cada 
estudiante en el proceso de desarrollo de sus estudios de Maestría así como la elaboración de su Investigación final de grado en un período récord de dieciséis meses, pues así estaba establecido en los acuerdos firmados.

La PUCP acogió, en total, a 108 becarios procedentes de, prácticamente, todo el territorio nacional. Según el acuerdo, las menciones elegidas entre las ofrecidas por nuestra Universidad para los estudios de Maestría fueron: Maestría en Educación, mención Currículo, Maestría en Enseñanza de las Matemáticas, menciones Primaria y Secundaria y la Maestría en Integración e Innovación educativa de las Tecnologías de la Información y de la Comunicación. Un total de 43 becarios optaron por seguir la Maestría en Currículo, 28 eligieron la Maestría en Enseńanza de las Matemáticas y 37 la Maestría en Integración e Innovación educativa de las Tecnologías de la Información y de la Comunicación (Pronabec, 2014).

El sello de la universidad se vio reflejado desde el primer momento en que se inició el programa (setiembre de 2014), pues se desarrolló una estrategia exitosa de adaptación al sistema universitario tan particular como el que tiene la PUCP. Se desarrollaron actividades de integración y de acercamiento a los diferentes servicios de la universidad. Desde un primer momento, se buscó que los estudiantes becarios se sintieran parte de la comunidad universitaria, compartiendo entre sí y con los demás miembros, actividades cotidianas, como el uso de bibliotecas, actividades culturales, deportivas, recreativas, comedores y demás servicios. Esto ayudó, en gran medida, para que los alumnos se desenvolvieran de manera natural en un ámbito, en principio, totalmente ajeno al que estaban habituados pero que, muy pronto, se hizo familiar. A medida que pasaron los meses, esta adaptación fue, prácticamente, total y se acoplaron al sistema de estudio y de investigación académica, teniendo como resultado, la graduación exitosa del $100 \%$ de los becarios en el tiempo previsto según el acuerdo firmado entre el Pronabec y la Universidad.

Si bien es cierto, fue el Estado quien financió completamente los costos de formación y derechos académicos, la PUCP comprometió varias prestaciones y beneficios adicionales a las estipuladas en el convenio marco interinstitucional firmado. En ese sentido, muy aparte de lo que estaba acordado, cada programa de Maestría dedicó una serie de mecanismos de apoyo suplementarios y adicionales para optimizar los procesos de aprendizaje de los estudiantes becarios. Se organizaron, por ejemplo, talleres de reforzamiento y nivelación en inglés, destinados a aquellos que presentaban dificultades, con el fin de conseguir que todos los becarios se encuentren en condiciones de lograr la acreditación en el nivel intermedio en Comprensión Lectora (requisito fundamental para la obtención del Grado). 
Asimismo, se programaron horas adicionales de asesorías académicas para la elaboración de las investigaciones finales de grado para, de esa forma, asegurar la meta de graduación fijada para diciembre de 2015. De igual manera, se organizaron talleres especiales de capacitación en el uso de herramientas informáticas para la interpretación de los datos obtenidos en la aplicación de los instrumentos de evaluación. Se llevaron a cabo talleres de manejo del programa estadístico SPSS (investigaciones cuantitativas) y de Atlas TI (investigaciones cualitativas). Se desarrollaron talleres de oratoria, sustentación y argumentación académica, talleres de imagen personal y etiqueta social, redacción académica, uso de las normas APA y talleres complementarios relacionados con las áreas de estudio de cada Maestría (Currículo, Tecnologías y Matemáticas) e, inclusive, actividades deportivas. Los alumnos becarios también participaron de todos los eventos programados tanto dentro del campus universitario como de otras instituciones y universidades. De esa manera, fueron activamente convocados y concurrieron a congresos, seminarios, conferencias, conversatorios y demás encuentros académicos que perfeccionaron sus conocimientos.

Por todo ello, no es aventurado mencionar que el gran aporte formativo que la universidad confirió a este grupo de becarios es difícil de cuantificar, aunque, efectivamente, y en función de los resultados, marcó un antes y un después en lo que se refiere a formación general y académica de los becarios.

\section{El proceso de elaboración de la inVestigación de MaESTría}

Como parte del acuerdo firmado entre el Pronabec y los alumnos becarios, estos se comprometían a realizar su investigación final de Maestría estableciendo como base y punto de partida la realidad particular que presenta su zona laboral de procedencia con el objetivo por un lado, de concientizar a cada becario con el contexto en el que se desarrollaban profesionalmente y por el otro de contribuir a través de los contenidos asimilados durante los estudios de Maestría a la mejora de las condiciones educativas de su zona de origen. De esta manera se buscaba crear una vez finalizada la Maestría, una red de docentes comprometidos con el desarrollo educativo del país. En ese contexto, la Universidad proporcionó todas las facilidades para el desarrollo del trabajo de campo reprogramando las clases y asesorías que estaban previstas para que los alumnos pudiesen efectuar durante el período de quince días, el proceso de aplicación de instrumentos de evaluación en sus localidades. A través de cartas de presentación y coordinaciones adicionales con las diversas UGEL y con los directivos de las instituciones educativas se logró articular el trabajo 
de investigación con las actividades curriculares en los colegios, asegurando que se otorguen a los estudiantes becarios las oportunidades y los ambientes apropiados para aplicar sus instrumentos sin dificultad.

\section{Metas y logros de la Beca Docente}

La convocatoria para el concurso de Beca Docente se realizó, a nivel nacional, a través de los organismos locales del Ministerio de Educación (Direcciones Regionales y Unidades de Gestión Educativas Locales). Se aplicaron evaluaciones descentralizadas en tres ciudades: Arequipa, Chiclayo y Lima. El sistema evaluativo consistió en: entrevista personal y pruebas escritas (tipo objetivo y redacción de un ensayo) (Pronabec, 2014).

Los aspirantes a la beca tenían la posibilidad de elegir la universidad a la que deseaban postular. Las universidades por su parte, se encargaron de planificar el proceso de admisión cumpliendo con las bases estipuladas en el Convenio marco, así como con el cronograma establecido y acordado (Pronabec, 2014).

Se cubrieron un total de 564, becas dentro de las cuales, 328 becarios procedían de las zonas urbanas, 166 de zonas rurales, 60 de zonas urbano marginales y 10 eran provenientes de zonas de frontera (Pronabec, 2014).

La Beca Docente, en ese sentido, promovió la inclusión de todos los sectores sociales de diversas regiones del país. De igual manera, brindó igualdad de oportunidades tanto para hombres como para mujeres y se consiguió una cifra equitativa para cada uno: 254 varones (45\%) y 310 mujeres (55\%) (Pronabec, 2014).

Las actividades académicas en las universidades integrantes del convenio se iniciaron de manera oficial en el mes de septiembre de 2014, casi de inmediato se detectaron algunos aspectos en la actitud y aptitud de los nuevos estudiantes que se creyó por conveniente trabajar con especial atención. La nueva condición de alumnos en un posgrado universitario para muchos de los becarios fue una etapa de adaptación lenta y no exenta de diversas dificultades. Pasadas unas semanas, un buen número de becarios evidenciaron cuadros de estrés, debido a la presión académica de los estudios, hecho que fue originado, en parte, porque, para ellos, el sistema formativo universitario de la PUCP era muy riguroso. El convenio interinstitucional contemplaba, además, la formación intensiva en el idioma inglés, de dos horas diarias, lo cual acrecentaba los niveles de presión académica en los estudiantes, teniendo en consideración que el grado de conocimiento de inglés que presentaba el grupo de becarios era bastante básico. Se sumaba a ello el hecho de que no habían recibido, anteriormente, cursos de metodología de trabajo intelectual ni formación previa en 
investigación, todo lo cual, en gran medida, dificultaba la adaptación grupal al ritmo de trabajo académico de la universidad. Es por ello, que se dispuso la organización de sesiones de tutoría enfocadas en metodología de estudio y trabajo intelectual, para ayudar a planificar de manera eficiente su aprendizaje diario, cumplir adecuadamente con las actividades propuestas y no generar ansiedad ni nerviosismo que, a su vez, derivaran en alguna sensación de frustración y fracaso en los estudiantes.

De la misma manera, los estudios universitarios de posgrado generaron, en los estudiantes, niveles de estrés y ansiedad. Otro aspecto significativo, dentro de la adaptación a un sistema nuevo de formación, fue encontrarse en un ambiente de estudio desconocido con sus propias normas y reglas institucionales, lo que, produjo, en muchos de los becarios, cierta resistencia al cambio. No obstante, progresivamente, fueron adoptando estas reglamentaciones como habituales y naturales, comprendiendo su sentido y propósito hasta conseguir, de esa manera, un estado de convivencia armónica propia de la comunidad universitaria. Aspectos esenciales como, por ejemplo, la falta de puntualidad, la desatención en el cumplimiento de los plazos, el desconocimiento de la formalidad en la presentación de trabajos, entre otras, fueron atendidos con prontitud desde el Programa de Maestría y se pusieron en marcha acciones concretas para corregir estos inconvenientes. En este sentido, como un valor agregado, se planificaron talleres y capacitaciones para desarrollar, en los becarios, aptitudes y actitudes relacionadas con el desarrollo personal, coaching, liderazgo, competencias sociales y de imagen personal, más allá de los conocimientos académicos.

Con el propósito de contribuir al mejor manejo del estrés y favorecer la camaradería entre los estudiantes, se organizaron también jornadas deportivas de fútbol y vóley semanales, al finalizar las actividades académicas en coordinación con el área de Deportes de la universidad, que proporcionó los permisos respectivos para el uso de los espacios deportivos del campus. La práctica del deporte favoreció, en gran medida, no solo el manejo del estrés y la mayor unidad grupal, sino, también, la mejora en la salud de los becarios, pues, en las revisiones médicas periódicas que se aplican a los estudiantes, se detectaron un menor número de problemas de salud como hipertensión, arritmias cardíacas, gastritis, migrańas, insomnio, entre otras dolencias.

$\mathrm{Si}$ bien es cierto que estos avances y progresos obtenidos por los estudiantes becarios durante su formación académica han tenido un nivel de repercusión e impacto positivo en ellos, como profesionales de la educación, y en el beneficio de la sociedad educativa, es cierto, también, que, al ser una beca de dedicación exclusiva que representó el desplazamiento geográfico de los estudiantes para 
alojarse durante 16 largos meses en una localidad diferente, alejados de su comunidad y de sus familias, pueden generar un sentimiento de desarraigo cultural que antes que desarrollar en ellos un compromiso real por trabajar en su localidad, los pueda alejar progresivamente de los propósitos y de los ideales primigenios que se trazaron al postular a la beca y que motivaron el esfuerzo de trasladarse. En otros casos, puede provocar un involuntario rechazo a su entorno cotidiano y buscar, entonces, nuevas oportunidades laborales en las grandes ciudades, desatendiendo, de esta manera, el compromiso asumido con el Ministerio de Educación para ejercer el efecto generador y multiplicador con su comunidad educativa de origen.

Con el fin de evitar el potencial desarraigo cultural derivado del prolongado alejamiento de los estudiantes de sus zonas de origen, se entendió que, para futuros proyectos, sería recomendable que los estudios de Maestría no representen, para los estudiantes, una permanencia constante ni prolongada en la ciudad donde se encuentre la universidad sino, más bien, experimentar con sistemas más flexibles de formación. Por ejemplo, en futuros programas se podrían diseñar sistemas formativos semipresenciales. De esa manera, podrían desarrollarse pasantías temporales en las universidades y completar los estudios utilizando entornos virtuales de aprendizaje, pero desde su misma localidad de origen, de tal forma que los estudiantes no pierdan ese contacto permanente con la realidad educativa de su localidad y desarrollen, así, actitudes de compromiso más firmes que se mantengan a largo plazo y que se conviertan en proyectos de desarrollo educativos sostenibles en el tiempo.

Pese a todo, la Beca Presidente de la República se constituyó en una experiencia de trabajo correctamente coordinada y articulada entre el sector privado y el Estado. Generalmente, se considera que es difícil conciliar las naturales diferencias existentes entre la gestión pública y la gestión privada, tomando en consideración que los procesos y procedimientos administrativos difieren de un sector a otro, en este caso; no obstante, se llegaron a aprobar y a adoptar muy importantes acuerdos interinstitucionales que ciertamente contribuyeron al mejor desarrollo de la maestría.

El esfuerzo desplegado por la universidad se ha visto reflejado en los buenos resultados obtenidos con este grupo. Los estudiantes, durante su formación académica, han incorporado diversos tipos de herramientas que, definitivamente, les han permitido ampliar su panorama profesional, lo cual ha significado un incremento de la autoestima personal y académica. De un perfil de ingreso de docentes vinculados con un perfil educativo de tipo convencional se pasó, al finalizar los estudios de Maestría, a un perfil de egreso orientado, primordialmente, a la Investigación y al desarrollo de proyectos. 
Se ha producido un gran salto cualitativo en el desempeño personal y profesional que es preciso destacar. Muchos de los graduados de las Maestrías, en la actualidad, se encuentran ejerciendo diversas funciones en instituciones educativas, en sus regiones de origen, no solo en instituciones de Educación Básica de donde provienen, sino que, también, se desempeñan como docentes universitarios o desarrollando labores de dirección administrativa en las diversas dependencias educativas descentralizadas.

Por otro lado, los altos niveles de cohesión e integración que se lograron entre el grupo de becarios es, también, un elemento digno de ponderar dentro de todo el conjunto de logros alcanzados. Esto se vio demostrado en la conformación de un grupo de trabajo que surgió de manera natural al formarse el grupo Compromiso Perú, una asociación de profesionales educadores reunidos en torno a un mismo objetivo: el desarrollo educativo y social del Perú. Esta iniciativa académica y profesional se organizó por zona geográfica según la localidad de origen de cada becario, de esta manera, los docentes becarios se agruparon en tres regiones definidas: costa, sierra y selva, para trabajar de manera conjunta y coordinada en proyectos de desarrollo educativo en sus respectivas regiones. Cabe destacar que esta forma de agrupamiento comprendía a las otras dos menciones de Maestría: Currículo y Enseñanza de las Matemáticas, lo cual amplió, aún más, el radio de acción de la asociación Compromiso Perú.

\section{Conclusiones}

La alianza público-privada de la Beca Docente, en su primera y hasta ahora única edición (2014-2015), ha sido cumplidamente beneficiosa y se han obtenido resultados auspiciosos y satisfactorios. Es evidente que esta experiencia está ya transfiriendo muy buenos frutos a mediano y largo plazo, en tanto ha contribuido, en gran medida, a la conformación de un grupo de profesionales competentes y comprometidos a compartir sus capacidades y talentos con sus comunidades, con sus entornos profesionales y con el avance científico del país; no obstante, es necesario, también, que estos esfuerzos continúen, se extiendan y reediten para seguir perfeccionando la calidad académica y profesional de los docentes y, de esta manera, favorecer la conformación de una efectiva cultura meritocrática de la carrera docente por la cual el ejercicio profesional del educador sea puesto en el valor cultural, social, ético y humano que verdadera y legítimamente le corresponde. 


\section{REFERENCIAS BIBLIOGRÁFICAS}

Claramunt, S. (2002). Orígenes del mundo universitario: de los studia a la universitas. En Joan J. Busqueta Juan Pemán (coord.), Les universitats de la Corona d'Aragó, ahir i avui (pp. 27-51). Barcelona: Pòrtic.

De la Calle, C. (18 de febrero de 2017). La formación de la responsabilidad social en la universidad. Recuperado de http://search.proquest.com/openview/dc1c f6b12fb583f2b26f33ea438919b8/1?pq-origsite=gscholar

De la Cuesta, M. (2010). Responsabilidad Social Universitaria: Del discurso simbólico a los desafios reales (Universidad da Coruña, ed.). La Coruña: Netbiblo. Recuperado de https://books.google.com.pe/books?hl=es\&lr=\&id=T-ZjJ SnvFaYC\&oi=fnd\&pg=PA3\&dq=responsabilidad + universitaria + conce pto\&ots=NubQ7Qxc9F\&sig=9PdiFuk5tI5IPALe8CkcDYqVctI\#v=one page $\& q=$ responsabilidad $\% 20$ universitaria $\% 20$ concepto $\& f=$ false

De la Red, N. (2009). Necesidades emergentes y responsabilidad social universitaria (U. de Alicante, ed.). Alternativas. Cuaderno de Trabajo Social (16).

Gasca-Pliego, E. y Olvera, J. (mayo-agosto de 2011). Construir ciudadanía desde las universidades, responsabilidad social universitaria y desafíos ante el siglo XXI. Convergencia, 18(56). Recuperado de http://www.scielo.org. $\mathrm{mx} /$ scielo.php?script=sci_arttext\&pid=S1405-14352011000200002

Martí Noguera, J. y Martí Vilar, M. (2013). Una década de Responsabilidad social universitaria en Iberoamérica. Revista Española del Tercer Sector, (25), $145-161$.

Martínez de Carrasquero, C. (2008). La responsabilidad social universitaria como estrategia de vinculación con su entorno social. Fronesis, 15(3).

Martinez, M. (junio de 2010). Aprendizaje servicio y construcción de ciudadanía activa en la universidad: la dimensión social y cívica de los aprendizajes académicos. En M. Martínez, Aprendizaje servicio y responsabilidad social (p. 218). Barcelona, Espańa: Octaedro - ICE. Recuperado de http://www. octaedro.com/pdf/110173Aprendizaje.pdf\#page $=25$

Pronabec (2014). Beca Presidente de la República. Bases del concurso 2014-I (Maestría o Doctorado internacional). Recuperado de http://www.pronabec.gob.pe/inicio/becas/descargas/bpresidente_bases.pdf

PUCP (2017). Responsabilidad social. Recuperado de http://www.pucp.edu.pe/ la-universidad/responsabilidad-social/

Vallaeys, F. (2017). ¿Qué es la responsabilidad social universitartia? Recuperado de http://creasfile.uahurtado.cl/RSU.pdf 